\title{
Editorial
}

\section{Advances in Geant4 applications in medicine}

Sebastien Incertia ${ }^{\mathrm{a} b},{ }^{*}$, Jeremy M.C. Brown ${ }^{\mathrm{c}}$, Susanna Guatelli ${ }^{\mathrm{d}}$

${ }^{a} C N R S, I N 2 P 3, C E N B G$, UMR 5797, Gradignan, France

'Université de Bordeaux, CENBG, UMR 5797, Gradignan, France

${ }^{\mathrm{C}}$ Radiation Science and Technology, Delft University of Technology, Delft, The Netherlands

${ }^{\mathrm{d} C e n t r e ~ F o r ~ M e d i c a l ~ R a d i a t i o n ~ P h y s i c s, ~ U n i v e r s i t y ~ o f ~ W o l l o n g o n g, ~ W o l l o n g o n g, ~ A u s t r a l i a ~}$

* Corresponding author at: CNRS, IN2P3, CENBG, UMR 5797, Gradignan, France

E-mail address: incerti@cenbg.in2p3.fr(S. Incerti).

The 3rd Geant4 International Conference at the Physics-Medicine-Biology Frontier, 29-31 October 2018, was organized in Bordeaux, France, by the Centre d'Etudes Nucléaires de Bordeaux-Gradignan (http://www.cenbg.in2p3.fr), a CNRS/IN2P3 and Bordeaux University research laboratory. The conference was chaired by Sébastien Incerti (CNRS/IN2P3, Bordeaux, France), with the support of two co-chairs Jeremy M. C. Brown (TUDelft, Delft, The Netherlands) and Susanna Guatelli (Wollongong University, Wollongong, Australia). The event gathered 111 participants from around the world. All participants had the opportunity to present their work in plenary oral sessions. This is the third iteration of the conference series (http://geant4.in2p3.fr) that was initiated in Bordeaux in 2005 under the auspices of the Geant4 collaboration (http://geant4.org). This series of conferences aims to gather Geant4/Geant4-based tool developers and users, in order to discuss Geant4 recent advances in medical physics and related topics (i.e. radiobiology).

Following an initiative started for the 2013 iteration of the conference [1], participants were proposed to submit regular papers for peer-review in a quality journal. For this conference, a Special Issue of Physica Medica, the European Journal of Medical Physics, was fully dedicated to the event, and a number of research projects presented during the conference were published.

The conference included overview talks on the most recent developments of the Geant4 toolkit [2-4] by Asai, on standard electromagnetic and hadronic physics by Ivanchenko, and on low/very low energy electromagnetic physics including Geant4-DNA [5-8] (http://geant4dna.org) by Incerti. Eight topical sessions were organized, ranging from developments of more accurate description of fundamental particle-matter physical interactions, to a variety of medical physics applications, spanning sub-cellular dosimetry to patient radiotherapy treatments. Geant4-based tools, such as GAMOS [9] (http://fismed.ciemat.es/GAMOS), GATE [10-13] (http://www.opengatecollaboration.org), and TOPAS [14] / TOPASnBio (http://www.topasmc.org) were also presented, notably outlining the ease to which they provide access for users to Geant4 simulations. 
The first two sessions were dedicated to the most recent developments of Geant4. Novak presented a new version of the Goudsmit-Saunderson multiple scattering model leading to significant improvement of electron and positron transport. Ivanchenko described a new data set of hadronic cross sections for the transport of low energy neutrons and light ions. Hajima and Omer proposed a new extension for polarized MeV photons of their elastic scattering model, soon released in Geant4. Bakr et al. presented a new accurate set of K, $\mathrm{L}$ and $\mathrm{M}$ shell ionisation cross sections for PIXE simulations with protons and alphas, originating from the ANSTO database. They also benchmarked the performance of Geant4 for the simulation of Auger cascades, in comparison to other Monte Carlo simulation. For very low energy simulations in liquid water with Geant4-DNA (http://geant4-dna.org), Shin et al. introduced a new discrete elastic scattering model for electrons calculated using the ELSEPA code. In addition, Dominguez-Munoz et al. presented first calculations of discrete ionisation by incident protons above $100 \mathrm{MeV}$. Regarding cross sections for other materials in Geant4-DNA, Mouawad et al. presented total cross sections for ionisation of biomolecules by incident electrons, and Nettelbeck et al. described the implementation of nitrogen and propane cross sections for incident electrons and ions. Moving up to larger scale simulations, Perales et al. explained the determination of water mean ionisation potential for protons and helium beams. Sécher et al. presented the benchmarking of Geant4 hadronic models from proton induced reaction on a lithium target, and Bolst et al. validated fragmentation models for therapeutic ${ }^{4} \mathrm{He}$ beams including differences with respect to experimental data. Mancini-Terracciano et al. [15] then described the first coupling of Geant4 to SMF and BLOB models for further improvement of Geant4 hadronic models. Finally, Caccia et al. gave an overview of Geant4 advanced examples for medical applications.

The next two sessions focused on imaging and instrumentation simulations. The first set of talks focused mainly on proton beams. Bongrand et al. presented Geant4 simulation of the Large area pixelized detector (LAPD) for in-beam ballistic control of hadrontherapy treatments, as well as validation against $65 \mathrm{MeV}$ proton data. Taylor et al. discussed the Geant4 simulation of a novel prompt gamma ray detection system for range verification in proton therapy and empirical validation. Allport et al. presented prompt-gamma ray production measurements at the Birmingham MC40 cyclotron and comparison with Geant4 predictions. Etxebeste et al. introduced an extension to the GATE platform to model Compton camera systems. Sarrut et al. proposed a generic approach for the learning of Monte Carlo detector response in SPECT with artificial neural networks. A series of talks addressing microdosimetry were then presented: Bolst et al. discussed the performance of Geant4 for the simulation of solid-state Silicon-on-insulator microdosimetry in heavy ion therapy; Prieto-Pena et al. showed microdosimetry measurements performed in a cyclotron beamline using new 3D-microdetectors and verifications using GATE and FLUKA; finally, Peracchi et al. described Geant4 simulations of the radiation environment aboard the International space station as well as simulations of a 3D "mushroom" microdosimeter, allowing a reliable evaluation of astronaut's absorbed dose. Collamati and colleagues described the Geant4 simulation of a CMOS sensor for beta particles detection and its validation and Gillet et al. discussed the validation of a new detector for CT dosimetry using GATE. Lansonneur et al. [16] then described the GATE simulation and validation of dose obtained from a prototype of electron beam accelerator in the perspective of the interpretation of radiobiological experiments in FLASH irradiation. On a similar topic, Solovev 
et al. presented Geant4 simulations allowing to set up radiobiological experiments with carbon ions at the U-70 synchrotron in Protvino, Russia. Michelet et al. [17] presented Geant4 simulations for 3D proton imaging of microscopic samples, and Sarno et al. described cone-beam breast CT also using Geant4. Beaudoux et al. [18] compared Geant4 electromagnetic physics lists for the simulation of phase-contrast mammography (in the context of the "XPulse" project) and finally Sosnovec described the possibilities to treat cancer by photodynamic therapy and related improvements.

The fifth session was exclusively dedicated to the investigation of the usage of gold nanoparticles (GNPs) in combination with ionising radiation. Sakata et al. [19] presented new discrete Geant4-DNA models to simulate electron interactions in GNPs. Villagomez-Bernabe presented dose enhancement simulations with TOPAS in lung cancer treatment using small clusters of GNPs activated by a $6 \mathrm{MeV}$ True Varian linac. Engels and colleagues evaluated Geant4-DNA physics models for GNP radio-enhancement in a clinical KV X-ray radiotherapy field, including influence on cell survival, and Hespeels et al. presented the use of the binary encounter approximation model to estimate absorbed dose and radiolysis yield of GNPs in protontherapy. Finally, Li et al. [20] presented a comparison of simulations of physical radiation quantities for GNPs irradiated by X-rays using a variety of Monte Carlo codes and the assessment of their potential use for targeted breast cancer treatment

Session six focused on protons and hadrons beams. Lens et al. presented the simulation of daily treatment monitoring with TOPAS from prompt gamma emission profiles detected during pencil beam protontherapy. Bagulya and Ivanchenko introduced in Geant4 a new ionisation model for ions based on a parameterisation of the relativistic theory of J. Linhard and A.H. Sorensen for ion energy loss. Then Burigo presented the evaluation of the impact of an in-line magnetic field on intensity-modulated proton therapy plans thanks to TOPAS and investigated a strategy for plan delivery adaption. Baumann and colleagues also used TOPAS to analyse the effects of Bragg curve degradation due to lung parenchyma in treatment planning. Grevillot et al. presented the new GATE-RTion independent dose calculation system for ion beam therapy and first validation for patient specific quality assurance, and Bolsa-Ferruz et al. further presented GATE for light ion beam dosimetry. Guardiola and colleagues presented dosimetric studies to guide the preclinical trials in proton minibeam radiotherapy using GATE. Lentz et al. described a hybrid method calculating linear energy transfer for intensity modulated proton therapy from Geant4 simulations. Rucinski et al. discussed the implementation and validation of a clinical proton beam model in GATE and in the GPU-accelerated Monte Carlo code Fred for quality assurance and detector development applications. Grzanka et al. investigated the radiobiological effect of nuclear fragments in proton radiotherapy using Geant4. Ali et al. used GATE to evaluate the biological effectiveness of $65 \mathrm{MeV}$ therapeutic proton beams, and Petringa et al. presented Geant4 simulations and experiments for the study of relative biological effectiveness of a clinical eye proton therapy beam.

The seventh session was dedicated to the presentation of user-friendly tools based on Geant4. Perl and colleagues presented an overview of TOPAS underlining its ease of use and extensibility. Arce et al. described the GAMOS framework and its main features. Gutierrez et al. proposed a code sharing effort for Monte Carlo beam models in radiotherapy. Nevay et al. presented the beam delivery simulation tool BDSIM for start-to-end accelerator 
simulations. Kurup et al. [21] described the simulation of a radiobiology facility for the clinical application of particles interdisciplinary collaboration. Abhyankar et al. [22] then presented performance analysis of the TOPAS code for a FPGA-based reconfigurable computing implementation perspective. Adamczyk et al. described a Geant4-based flexible software toolkit for simulating the total dose produced by medical linear accelerators. Han et al. described their new ICRP adult mesh-type reference computational phantoms and their applications using Geant4, and Fois et al. presented CPOP, an open-source C++ cell population modeler combined to Geant4 simulations for radiation biology.

Sessions eight and nine focused on radiotherapy and clinical dosimetry. Malcolm and colleagues presented GATE simulations of ${ }^{177}$ Lu therapies towards a personalized patient biological dosimetry, followed by a general overview talk about the GATE platform by Maigne et al. Dezarn described image-based S voxel patient dosimetry in lung tissue following liver transarterial radioembolization using Geant4. Arbor et al. [23] then presented GATE simulation of breast cancer treatment for the evaluation of superficial dose calculations. Buonanno et al. [24] described a validated Geant4 study on rotational radiotherapy of breast cancer with a polyenergetic kilovoltage $X$-ray beam, and Pirozzi Palmese et al. introduced rotational radiotherapy of breast cancer with synchrotron radiation microbeams where Geant4 was used for dosimetry. Currell reviewed effects of nanoparticle-based systems towards clinical predictions and underlined the necessity for validated radiation chemistry models. Spiga et al. [25] presented Geant4 simulations for photon activation therapy with metallic compound in agreement with measurements. Shin and colleagues described Geant4 simulations of a kilovoltage irradiation system in the context of animal companion healthcare. Salas-Ramirez et al. developed a radiation transport model for bone marrow dosimetry based on tessellated volumes using GATE. Finally, Arce and Lagares showed a detailed study of Geant4 electromagnetic physics on the precision and speed for dose calculation of a gamma radiotherapy treatment.

The last two sessions focused on Geant4-DNA and included the largest number of oral presentations, underlining the importance of Geant4 mechanistic modelling capabilities at very low energy and small scale in biological medium. Sakata et al. [26] first presented a full integrated Geant4-DNA simulation chain for the prediction of early direct and indirect DNA damage in a cell nucleus model based notably on IRT radiolysis simulation. De la Fuente and colleagues further discussed the modelling of indirect radiation damage using Geant4-DNA, using in particular an atomistic resolution DNA geometrical model. Villagrasa et al. presented a European effort for the benchmarking of Monte Carlo calculations for micro- and nanodosimetry simulations, detailing the contribution of cross-sections to the simulation results' uncertainty. Tang et al. discussed the influence of chromatin compaction on simulated early radiation-induced DNA damage using Geant4-DNA. Ramos-Mendez et al. introduced TOPAS-nBio capabilities for an easier experimental validation of Geant4-DNA radiolysis simulations. Stepan and colleagues presented the simulation of radiation-induced plasmid DNA damage, including a library of plasmid configurations that can be used in Geant4-DNA. Torfeh et al. described the correlation of Geant4-DNA microdosimetric simulations with in cellulo radiation-induced spatiotemporal dynamics of GFP-tagged DNA repair protein measured at CENBG, and Tany et al. described the investigation of radionuclide loaded nano-carrier induced DNA damage at the sub-cellular scale using Geant4-DNA. Kyriakou et al. [27] discussed the influence of track structure and condensed 
history physics models of Geant4 to nanoscale electron transport in liquid water. Okada et al. presented the porting of Geant4 physics and Geant4-DNA physics and chemistry to GPU in the context of the MPEXS initiative, reaching impressive computing performance. Petringa et al. introduced new methods for linear energy transfer and relative biological effectiveness calculation using Geant4. Smith et al. described biologically relevant predictions for proton beam therapy from in silico modelling, using in particular Geant4-DNA, and their relevance to treatment planning. Baratto-Roldan used Geant4-DNA for microdosimetry-based doseaverage linear energy transfer calculation for clinical proton beams. Thongjerm and Barlow described the study of energy distribution of particles at the same depth using Geant4-DNA simulations. Warmenhoven presented the DaMaRiS in silico model of DNA repair for the investigation of mechanisms in non-homologous end joining. Liu et al. presented the RADCEL integrated spatial and temporal stochastic model for simulating radiation transportation in cells. Finally, Desorgher et al. presented the first investigation of the radiotherapy FLASH effect using Geant4-DNA.

Three other manuscripts were submitted to the Special Issue and were recently published : one by Pettersen et al. [28] on the design optimization of a pixel-based range telescope for proton computed tomography, a study on silicon based microdosimetry for Boron Neutron Capture Therapy Quality Assurance by Vohradsky et al. [29] and a third manuscript by Lee et al. [30] on a cell-by-cell Monte Carlo simulation for assessing radiation- induced DNA double strand breaks.

In summary, this conference and its previous iterations $(2015,2013)$, as well as the three Monte Carlo international workshops for radiotherapy, imaging and radiation protection [31] that were organized at Wollongong University, Australia, bring unique occasions to gather Geant4 developers and users in a constantly growing interdisciplinary research domain at the frontier between Physics, Medicine and Biology. We hope to be able to continue the organization of such events, which regularly trigger new collaborations and contribute to the emergence of future developments and original applications of Geant4. Monte Carlo simulations have become over the years a golden standard for Medical Physics and the open source Geant4 toolkit is today undoubtedly a key actor.

\section{Acknowledgments}

The Guest Editors would like to thank the Editor in Chief Paolo Russo for making this Special Issue possible and for his constant support to Geant4 over the years. They also thank the Geant4 collaboration at the heart of all these research and development activities at the Physics-Medicine-Biology frontier. S. Incerti thanks the National institute of nuclear and particle physics (IN2P3) of the National centre for scientific research (CNRS), as well as Bordeaux University for their support. Finally, they congratulate Michel Maire (Annecy, France) for his outstanding contributions to Geant4 over the years. 

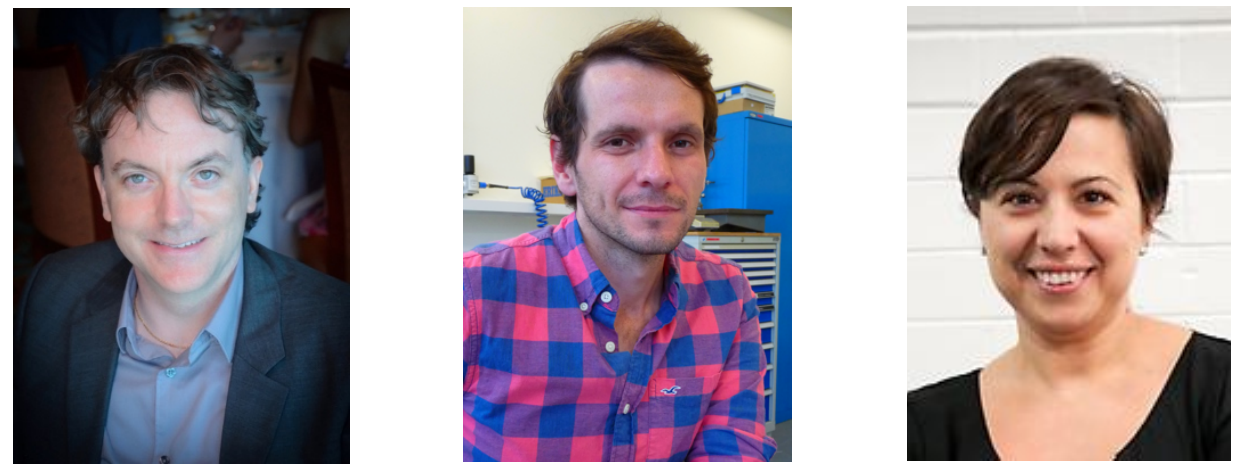

Left to right : S. Incerti, J. M. C. Brown, S. Guatelli

\section{References}

[1] Incerti S. Special section: selected papers from the Geant4 2013 international user conference at the physics-medicine-biology frontier. Physics in Medicine and Biology. 2014;59:7565.

[2] Agostinelli S, Allison J, Amako K, Apostolakis J, Araujo H, Arce P, et al. G4--a simulation toolkit. Nuclear Instruments and Methods in Physics Research Section A: Accelerators, Spectrometers, Detectors and Associated Equipment. 2003;506:250-303.

[3] Allison J, Amako K, Apostolakis J, Araujo H, Dubois PA, Asai M, et al. Geant4 developments and applications. Nuclear Science, IEEE Transactions on. 2006;53:270-8. [4] Allison J, Amako K, Apostolakis J, Arce P, Asai M, Aso T, et al. Recent developments in Geant4. Nuclear Instruments and Methods in Physics Research Section A: Accelerators, Spectrometers, Detectors and Associated Equipment. 2016;835:186-225.

[5] Incerti S, Baldacchino G, Bernal M, Capra R, Champion C, Francis Z, et al. The Geant4-DNA project. International Journal of Modeling, Simulation, and Scientific Computing.

2010;01:157-78.

[6] Incerti S, Ivanchenko A, Karamitros M, Mantero A, Moretto P, Tran HN, et al. Comparison of Geant4 very low energy cross section models with experimental data in water. Medical Physics. 2010;37:4692-708.

[7] Bernal MA, Bordage MC, Brown JMC, Davídková M, Delage E, El Bitar Z, et al. Track structure modeling in liquid water: A review of the Geant4-DNA very low energy extension of the Geant4 Monte Carlo simulation toolkit. Physica Medica. 2015;31:861-74.

[8] Incerti S, Kyriakou I, Bernal MA, Bordage MC, Francis Z, Guatelli S, et al. Geant4-DNA example applications for track structure simulations in liquid water: $A$ report from the Geant4-DNA Project. Medical Physics. 2018;45:e722-e39.

[9] Arce P, Ignacio Lagares J, Harkness L, Pérez-Astudillo D, Cañadas M, Rato P, et al. Gamos: A framework to do Geant4 simulations in different physics fields with an user-friendly interface. Nuclear Instruments and Methods in Physics Research Section A: Accelerators, Spectrometers, Detectors and Associated Equipment. 2014;735:304-13.

[10] Santin G, Strul D, Lazaro D, Simon L, Krieguer M, Martins MV, et al. GATE, a Geant4based simulation platform for PET integrating movement and time management. 2002 IEEE Nuclear Science Symposium Conference Record2002. p. 1325-9 vol.2. 
[11] Strulab D, Santin G, Lazaro D, Breton V, Morel C. GATE (geant4 application for tomographic emission): a PET/SPECT general-purpose simulation platform. Nuclear Physics B - Proceedings Supplements. 2003;125:75-9.

[12] Jan S, Santin G, Strul D, Staelens S, Assié K, Autret D, et al. GATE: a simulation toolkit for PET and SPECT. Physics in Medicine \& Biology. 2004;49:4543.

[13] Jan S, Benoit D, Becheva E, Carlier T, Cassol F, Descourt P, et al. GATE V6: a major enhancement of the GATE simulation platform enabling modelling of $C T$ and radiotherapy. Physics in Medicine \& Biology. 2011;56:881.

[14] Perl J, Shin J, Schümann J, Faddegon B, Paganetti H. TOPAS: An innovative proton Monte Carlo platform for research and clinical applications. Medical Physics. 2012;39:6818-37.

[15] Mancini-Terracciano C, Asai M, Caccia B, Cirrone GAP, Dotti A, Faccini R, et al.

Preliminary results coupling "Stochastic Mean Field" and "Boltzmann-Langevin One Body" models with Geant4. Physica Medica. 2019;67:116-22.

[16] Lansonneur P, Favaudon V, Heinrich S, Fouillade C, Verrelle P, De Marzi L. Simulation and experimental validation of a prototype electron beam linear accelerator for preclinical studies. Physica Medica. 2019;60:50-7.

[17] Michelet C, Li Z, Yang W, Incerti S, Desbarats P, Giovannelli J-F, et al. A Geant4 simulation for three-dimensional proton imaging of microscopic samples. Physica Medica. 2019;65:172-80.

[18] Beaudoux V, Blin G, Barbrel B, Kantor G, Zacharatou C. Geant4 physics list comparison for the simulation of phase-contrast mammography (XPulse project). Physica Medica. 2019;60:66-75.

[19] Sakata D, Kyriakou I, Tran HN, Bordage M-C, Rosenfeld A, Ivanchenko V, et al. Electron track structure simulations in a gold nanoparticle using Geant4-DNA. Physica Medica.

2019;63:98-104.

[20] Li WB, Belchior A, Beuve M, Chen YZ, Di Maria S, Friedland W, et al. Intercomparison of dose enhancement ratio and secondary electron spectra for gold nanoparticles irradiated by $\mathrm{X}$-rays calculated using multiple Monte Carlo simulation codes. Physica Medica.

2020;69:147-63.

[21] Kurup A, Pasternak J, Taylor R, Murgatroyd L, Ettlinger O, Shields W, et al. Simulation of a radiobiology facility for the Centre for the Clinical Application of Particles. Physica Medica. 2019;65:21-8.

[22] Abhyankar YS, Dev S, Sarun OS, Saxena A, Joshi R, Darbari H, et al. Monte Carlo Processing on a Chip (MCoaC)-preliminary experiments toward the realization of optimalhardware for TOPAS/Geant4 to drive discovery. Physica Medica. 2019;64:166-73.

[23] Arbor N, Gasteuil J, Noblet C, Moreau M, Meyer P. A GATE/Geant4 Monte Carlo toolkit for surface dose calculation in VMAT breast cancer radiotherapy. Physica Medica.

2019;61:112-7.

[24] Buonanno F, Sarno A, De Lucia PA, Di Lillo F, Masi M, Di Franco F, et al. Rotational radiotherapy of breast cancer with polyenergetic kilovoltage $X$-ray beams: An experimental and Monte Carlo phantom study. Physica Medica. 2019;62:63-72.

[25] Spiga J, Pellicioli P, Manger SP, Duffy JA, Bravin A. Experimental benchmarking of Monte Carlo simulations for radiotherapy dosimetry using monochromatic $\mathrm{X}$-ray beams in the presence of metal-based compounds. Physica Medica. 2019;66:45-54.

[26] Sakata D, Lampe N, Karamitros M, Kyriakou I, Belov O, Bernal MA, et al. Evaluation of early radiation DNA damage in a fractal cell nucleus model using Geant4-DNA. Physica Medica. 2019;62:152-7. 
[27] Kyriakou I, Ivanchenko V, Sakata D, Bordage MC, Guatelli S, Incerti S, et al. Influence of track structure and condensed history physics models of Geant4 to nanoscale electron transport in liquid water. Physica Medica. 2019;58:149-54.

[28] Pettersen HES, Alme J, Barnaföldi GG, Barthel R, van den Brink A, Chaar M, et al. Design optimization of a pixel-based range telescope for proton computed tomography. Physica Medica. 2019;63:87-97.

[29] Vohradsky J, Guatelli S, Davis JA, Tran LT, Rosenfeld AB. Evaluation of silicon based microdosimetry for Boron Neutron Capture Therapy Quality Assurance. Physica Medica. 2019;66:8-14.

[30] Lee BH, Wang CKC. A cell-by-cell Monte Carlo simulation for assessing radiation-induced DNA double strand breaks. Physica Medica. 2019;62:140-51.

[31] Guatelli S, Incerti S. Monte Carlo simulations for medical physics: From fundamental physics to cancer treatment. Physica Medica: European Journal of Medical Physics. 2017;33:179-81. 